Journal of Advanced Research in Name
Vol. 00 No. 0, pp. 1-9

October 2011

\title{
On Baer Invariants of Triples of Groups
}

\author{
Zohreh Vasagh, Hanieh Mirebrahimi, and Behrooz Mashayekhy* \\ Department of Pure Mathematics, Center of Excellence in Analysis on Al- \\ gebraic Structures, Ferdowsi University of Mashhad, P.O.Box 91775-1159, \\ Mashhad, Iran.
}

\begin{abstract}
In this paper, we develop the theory of Baer invariants for triples of groups. First, we focus on the general properties of the Baer invariant of triples. Second, we prove that the Baer invariant of a triple preserves direct limits of directed systems of triples of groups. Moreover, we present a structure for the nilpotent multiplier of a triple of the free product in some cases. Finally, we give some conditions in which the Baer invariant of a triple is a torsion group.
\end{abstract}

Keywords: Baer invariant; Triple of groups; Simplicial groups.

AMS subject classifications: 57M07, 20J05, 55U10

\section{Introduction and Motivation}

Throughout the article, let $\mathcal{V}$ be an arbitrary variety of groups defined by a set of laws $V$ and consider the functor $V(-)$ between groups which takes a group to its verbal subgroup. By a triple of groups $(G, M, N)$ we mean a group $G$ with two normal subgroups $N, M$. A homomorphism of triples $\left(G_{1}, M_{1}, N_{1}\right) \rightarrow\left(G_{2}, M_{2}, N_{2}\right)$ is a group homomorphism $G_{1} \rightarrow G_{2}$ that sends $M_{1}$ into $M_{2}$ and $N_{1}$ into $N_{2}$.

Finding a topological interpretation for algebraic concepts can be useful to solve some algebraic problems. The Schur multiplier, $M(G)$, of an arbitrary group $G$ can be described as the second integral homology $H_{2}(X)$ of a connected CW-space $X$, where $X$ has the fundamental group $G$ and contractible universal cover [8]. The Baer invariant of $G, \mathcal{V} M(G)$, has a similar topological interpretation. For this, consider the first homotopy group, $\pi_{1}(K . / V(K)$.$) , of the factor of a free simplicial resolution of the group G$ [6].

${ }^{*}$ Correspondence to: Behrooz Mashayekhy, Department of Pure Mathematics, Center of Excellence in Analysis on Algebraic Structures, Ferdowsi University of Mashhad, P.O.Box 91775-1159, Mashhad, Iran. Email: bmashf@um.ac.ir

http://www.i-asr.org 
G. Ellis [5] defined the Schur multiplier of a pair of groups $(G, N)$ denoted by $M(G, N)$, as a functorial abelian group whose principal feature is a natural exact sequence

$$
\begin{aligned}
\cdots \quad \rightarrow M(G, N) \rightarrow M(G) \rightarrow M(G / N) \\
\quad \rightarrow N /[N, G] \rightarrow G^{a b} \rightarrow(G / N)^{a b} \rightarrow 0 .
\end{aligned}
$$

The natural epimorphism $G \rightarrow G / N$ implies the following exact sequence of free simplicial groups

$$
1 \rightarrow \operatorname{ker}(\alpha) \rightarrow K \stackrel{\alpha}{\rightarrow} L . \rightarrow 1,
$$

where $K$. and $L$. are free simplicial resolutions of $G$ and $G / N$, respectively [6]. The authors in [10] extended the above notation to the Baer invariant of a pair of groups, $\mathcal{V} M(G, N)$, as the first homotopy group of the kernel of a map $\alpha_{v}, \pi_{1}\left(\operatorname{ker} \alpha_{v}\right)$, where $\alpha_{v}: K . / V(K.) \rightarrow L_{.} / V\left(L_{.}\right)$is induced from the simplicial map $\alpha$. Also the authors obtained the following long exact sequence

$$
\begin{aligned}
\cdots \quad & \rightarrow \mathcal{V} M(G, N) \rightarrow \mathcal{V} M(G) \rightarrow \mathcal{V} M(G / N) \\
& \rightarrow N /\left[N V^{*} G\right] \rightarrow G / V(G) \rightarrow(G / N) / V(G / N) \rightarrow 0 .
\end{aligned}
$$

The Schur multiplier of a triple was first studied by Ellis [5], who introduced it as a functorial abelian group $M(G, M, N)$ which fits into the following natural exact sequence

$$
\begin{aligned}
\cdots & \rightarrow M(G, M, N) \rightarrow M(G, N) \rightarrow M(G / M, M N / M) \\
& \rightarrow M \cap N /[M \cap N, G][M, N] \rightarrow N /[N, G] \rightarrow M N / M[N, G] \rightarrow 0 .
\end{aligned}
$$

In this article, we intend to extend the concept of the Schur multiplier of triples to the Baer invariant of triples of groups with respect to an arbitrary variety $\mathcal{V}$ using homotopy theory for simplicial groups and free simplicial resolutions of groups.

Definition 1.1. Consider the following natural commutative diagram of a triple of groups $(G, M, N)$

$$
\begin{array}{ccc}
G & \rightarrow & \frac{G}{N} \\
\downarrow & & \downarrow \\
\frac{G}{M} & \rightarrow & \frac{G}{M N}
\end{array}
$$

which implies the following commutative diagram of simplicial groups

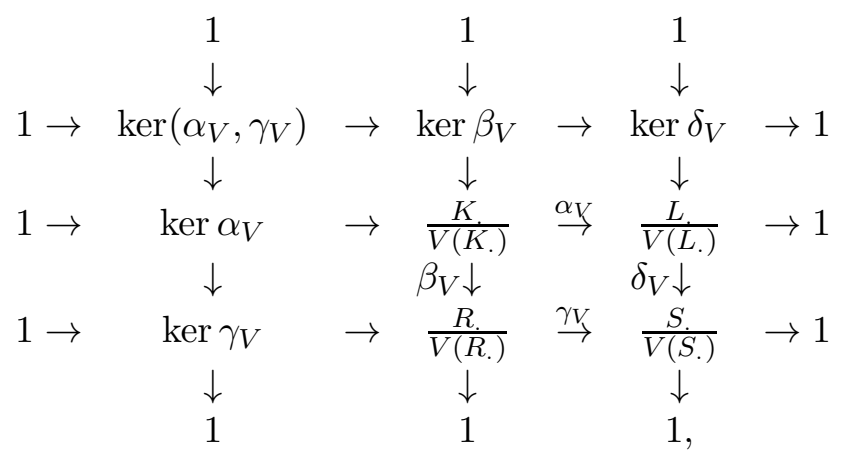


where $K, L . R$. and $S$. are free simplicial resolutions of $G, G / N, G / M$ and $G / M N$, respectively [1]. We define the Baer invariant of the triple $(G, M, N)$ with respect to a variety of groups $\mathcal{V}$ as follows

$$
\mathcal{V} M(G, M, N)=\pi_{1}\left(\operatorname{ker}\left(\alpha_{V}, \gamma_{V}\right)\right),
$$

where $\operatorname{ker}\left(\alpha_{V}, \gamma_{V}\right)$ is defined in diagram (1.1).

The article is organized as follows. In Section 2, we give a brief review for simplicial groups and some of their properties needed for the rest. In Section 3, First we give some useful general results for computation of Baer invariant of triple of groups. In particular, we obtain long exact sequences that contain Baer invariants of triples and pairs of groups. Second, we prove that the Baer invariant of a triple preserves direct limits of directed systems of triples of groups. Moreover, we study the behavior of $c$-nilpotent multiplier with respect to the free product in some cases. Finally, we obtain some conditions in which the $c$-nilpotent multiplier of a triple of groups is a torsion group.

\section{Preliminaries and Notation}

In this section we recall several concepts and results about the simplicial groups which will be needed in the sequel. A detailed can be found in [3] or [7].

A simplicial group $K$. is a sequence of groups $K_{0}, K_{1}, K_{2}, \ldots$ together with homomorphisms $d_{i}: K_{n} \rightarrow K_{n-1}$ (faces) and $s_{i}: K_{n} \rightarrow K_{n+1}$ (degeneracies), for each $0 \leq i \leq n$, such that the following conditions hold:

$$
\begin{aligned}
& d_{j} d_{i}=d_{i-1} d_{j} \quad \text { for } j<i \\
& s_{j} s_{i}=s_{i+1} s_{j} \quad \text { for } j \leq i \\
& d_{j} s_{i}= \begin{cases}s_{i-1} d_{j} & \text { for } j<i \\
i d e n t i t y & \text { for } j=i, i+1 \\
s_{i} d_{j-1} & \text { for } j>i+1\end{cases}
\end{aligned}
$$

A simplicial homomorphism $f: K$. $\rightarrow L$. is a sequence of homomorphisms $f_{n}: K_{n} \rightarrow L_{n}$, for each $n \geq 0$, such that $f \circ d_{i}=d_{i} \circ f$, and $f \circ s_{i}=s_{i} \circ f$, that is the following diagram commutes:

$$
\begin{array}{rrrrrr}
K_{n+1} & \stackrel{s_{i}}{\leftarrow} & K_{n} & \stackrel{d_{i}}{\rightarrow} & K_{n-1} \\
f_{n+1} \downarrow & & f_{n} \downarrow & & f_{n-1} \downarrow \\
L_{n+1} & \stackrel{s_{i}}{\leftarrow} & L_{n} & \stackrel{d_{i}}{\rightarrow} & L_{n-1} .
\end{array}
$$

From a simplicial group $K$. we can derive a chain complex $(N K, \partial)$ which is called the Moore complex as follows: $(N K)_{n}=\cap_{i=0}^{n-1} \operatorname{Kerd}_{i}$ and $\partial_{n}: N K_{n} \rightarrow N K_{n-1}$ to be the restriction of $d_{n}$.

Theorem 2.1. ([3]).

1. For every simplicial group $K$. the homotopy group $\pi_{n}\left(K_{\text {. }}\right)$ is abelian even for $n=1$. 
2. Every epimorphism between simplicial groups is a fibration.

3. Let $K$. be a simplicial group, then $\pi_{*}(K.) \cong H_{*}(N K$.$) .$

4. $H_{n}(N(K . \otimes L).) \cong H_{n}\left(N(K.) \otimes N\left(L_{.}\right)\right)$.

A simplicial group $K$. is said to be free if each $K_{n}$ is a free group and degeneracy homomorphisms $s_{i}$ 's send the free basis of $K_{n}$ into the free basis of $K_{n+1}$. A free simplicial resolution of $G$ consists of a free simplicial group $K$. with $\pi_{0}\left(K_{\text {. }}\right)=G$ and $\pi_{n}(K)=$.$o for n \geq 1$ (see [9]).

\section{Baer Invariants of Triples}

In this section, we study the behavior of Baer invariants of triples of groups. First, we give some basic and essential properties of the Baer invariant of a triple of groups.

Theorem 3.1. The Baer invariant of a triple of groups is a functor from the category of triples of groups to the category of abelian groups.

Proof. Let $f:\left(G_{1}, M_{1}, N_{1}\right) \rightarrow\left(G_{2}, M_{2}, N_{2}\right)$ be a homomorphism of triples of groups, functorial property of free simplicial resolutions yields the proof completed.

Theorem 3.2. Let $(G, M, N)$ be a triple of groups and $\mathcal{V}$ be an arbitrary variety of groups defined by a set of laws $V$. Then $\mathcal{V} M(G, M, N)$ satisfies in the following long exact sequences

$$
\begin{aligned}
\cdots & \rightarrow \mathcal{V} M(G, M, N) \rightarrow \mathcal{V} M(G, N) \rightarrow \mathcal{V} M(G / M, M N / M) \\
& \rightarrow \pi_{0}\left(\operatorname{ker}\left(\alpha_{V}, \gamma_{V}\right)\right) \rightarrow N /\left[N V^{*} G\right] \rightarrow M N / M\left[N V^{*} G\right] \rightarrow 0
\end{aligned}
$$

and

$$
\begin{aligned}
\cdots \quad & \rightarrow \mathcal{V} M(G, M, N) \rightarrow \mathcal{V} M(G, M) \rightarrow \mathcal{V} M(G / N, M N / N) \\
& \rightarrow \pi_{0}\left(\operatorname{ker}\left(\alpha_{V}, \gamma_{V}\right)\right) \rightarrow M /\left[M V^{*} G\right] \rightarrow M N / N\left[M V^{*} G\right] \rightarrow 0 .
\end{aligned}
$$

Proof. Consider Diagram (1.1) corresponding to triple of groups $(G, M, N)$. In [6] one can find the following isomorphisms

$$
\begin{aligned}
\pi_{0}\left(\operatorname{ker} \alpha_{v}\right) & \cong \frac{N}{[N V * G]} \\
\pi_{0}\left(\operatorname{ker} \gamma_{v}\right) & \cong \frac{M N}{N[M V * G]} \\
\pi_{0}\left(\frac{K}{V(K .)}\right) & \cong \frac{G}{V(G)} \\
\pi_{0}\left(\frac{R}{V(R .)}\right) & \cong \frac{G / M}{V(G / M)}
\end{aligned}
$$

$$
\begin{aligned}
\pi_{0}\left(\operatorname{ker} \beta_{v}\right) & \cong \frac{M}{[M V * G]} \\
\pi_{0}\left(\operatorname{ker} \delta_{v}\right) & \cong \frac{M N}{M[N V * G]} \\
\pi_{0}\left(\frac{L}{V(L .)}\right) & \cong \frac{G / N}{V(G / N)} \\
\pi_{0}\left(\frac{S .}{V(S .)}\right) & \cong \frac{G / M N}{V(G / M N)}
\end{aligned}
$$

The left column of Diagram (1.1) yields the long exact sequence of homotopy groups, using the various isomorphisms we can rewrite it in the group theory language as the exact sequence (3.1). Also the top row of Diagram (1.1) induces the other long exact sequence. 
Theorem 3.3. Let $G=M N$ and $\mathcal{V}$ be the variety of abelian group of exponent $q$. Then we have the following long exact sequence

$$
\begin{aligned}
\cdots & \rightarrow \mathcal{V} M(G, M, N) \rightarrow \mathcal{V} M(G, N) \rightarrow \mathcal{V} M(G / M) \\
& \rightarrow M \cap N / M \#_{q} N \rightarrow N /\left[N V^{*} G\right] \rightarrow G / M / V(G / M) \rightarrow 1,
\end{aligned}
$$

where $M \#_{q} N$ is the subgroup of $G$ generated by

$$
[m, n] t^{q} ; \quad m \in M, n \in N \text { and } t \in M \cap N .
$$

Proof. Barja and Rodriguez in [1] obtained $\pi_{0}\left(\alpha_{V}, \gamma_{V}\right) \cong M \cap N / M \#_{q} N$ which gives the result.

The long exact sequence of (3.1) implies the following theorem.

Theorem 3.4. The Baer invariant of a pair of groups is a special case of the Baer invariant of a triple of groups i.e. $\mathcal{V} M(G, M, M) \cong \mathcal{V} M(G, M) \cong \mathcal{V} M(G, G, M)$ for any group $G$ and any normal subgroup $M$.

Theorem 3.5. Let $\left\{\left(G_{i}, M_{i}, N_{i}\right)\right\}_{i \in I}$ be a directed system of triples of groups. Then

$$
\mathcal{V} M\left(\lim _{\longrightarrow} G_{i}, \lim _{\longrightarrow} M_{i}, \lim _{\longrightarrow} N_{i}\right) \cong \lim _{\longrightarrow} \mathcal{V} M\left(G_{i}, M_{i}, N_{i}\right)
$$

Proof. For any $i \in I$, let $K_{i}, L_{i}, R_{i}$. and $S_{i}$. be corresponding free simplicial resolutions of $G_{i}, G_{i} / N_{i}, G_{i} / M_{i}$ and $G_{i} / M_{i} N_{i}$, respectively. Assume the following diagram of simplicial groups

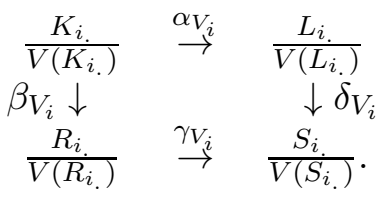

The authors in [10] proved that $\lim _{n} \pi_{n}\left(K_{i}\right) \cong \pi_{n}\left(\lim _{\longrightarrow} K_{i}\right)$, where $K_{i}$. are simplicial groups. Hence $\lim K_{i}, \quad \lim L_{i}, \quad \underline{\longrightarrow} R_{i}$, and $\underline{\lim } S_{i}$. are simplicial groups corresponding to $\underset{\lim }{\longrightarrow} G_{i}, \underset{\lim }{\rightarrow} G_{i} / N_{i}, \overrightarrow{\lim } G_{i} / \overrightarrow{M_{i}}$ and $\underset{\lim }{\longrightarrow} \overrightarrow{G_{i}} / M_{i} N_{i}$, respectively. Also, the authors [11, Theorem 2.7] proved that $\underset{\lim }{\longrightarrow}\left(\operatorname{ker} \alpha_{V_{i}}\right) \cong \operatorname{ker} \underset{\longrightarrow}{\longrightarrow}\left(\alpha_{V_{i}}\right)$ and similarly $\stackrel{\lim }{\longrightarrow}\left(\operatorname{ker} \gamma_{V_{i}}\right) \cong$ ker $\underset{\lim }{\longrightarrow}\left(\gamma_{V_{i}}\right)$. Now consider the following commutative diagram:

$$
\begin{aligned}
& 1 \rightarrow \operatorname{ker}\left(\left(\lim _{\downarrow} \alpha_{V_{i}}, \underset{\downarrow}{\lim } \gamma_{V_{i}}\right)\right) \rightarrow \underset{\downarrow}{\operatorname{ker}} \underset{\downarrow}{\lim }\left(\gamma_{V_{i}}\right) \rightarrow \operatorname{ker} \underset{\downarrow}{\lim }\left(\alpha_{V_{i}}\right) \rightarrow 1 \\
& 1 \rightarrow \quad \stackrel{\lim }{\longrightarrow}\left(\operatorname{ker}\left(\alpha_{V_{i}}, \gamma_{V_{i}}\right)\right) \quad \rightarrow \quad \stackrel{\lim }{\longrightarrow}\left(\operatorname{ker} \gamma_{V_{i}}\right) \rightarrow \underline{\lim }\left(\operatorname{ker} \alpha_{V_{i}}\right) \rightarrow 1 .
\end{aligned}
$$

Similar to the proof of [11, Theorem 2.7], the Five Lemma implies that

$$
\stackrel{\lim }{\longrightarrow}\left(\operatorname{ker}\left(\alpha_{V_{i}}, \gamma_{V_{i}}\right)\right) \cong \operatorname{ker}\left(\left(\lim _{\longrightarrow} \alpha_{V_{i}}, \lim _{\longrightarrow} \gamma_{V_{i}}\right)\right)
$$

Since homotopy groups of simplicial groups commute with direct limits [10], the following isomorphisms hold

$$
\begin{aligned}
& \mathcal{V} M\left(\lim _{\longrightarrow} G_{i}, \lim _{\longrightarrow} M_{i}, \lim _{\longrightarrow} N_{i}\right) \cong \pi_{1}\left(\operatorname{ker}\left(\lim _{\longrightarrow} \alpha_{V_{i}}, \lim _{\longrightarrow} \gamma_{V_{i}}\right)\right) \\
& \cong \pi_{1}\left(\overrightarrow{\lim }\left(\overrightarrow{\operatorname{ker}}\left(\alpha_{V_{i}}, \gamma_{V_{i}}\right)\right)\right) \quad \cong \underset{\lim }{\longrightarrow} \vec{M}\left(G_{i}, M_{i}, N_{i}\right) \text {. }
\end{aligned}
$$


Now we study the behavior of Baer invariants of triples of free products.

Theorem 3.6. Let $\left(G_{i}, M_{i}, N_{i}\right)$ be triples of groups, for $i=1,2$. Then the following isomorphism holds

$$
M\left(G_{1} * G_{2},\left\langle M_{1} * M_{2}\right\rangle^{G_{1} * G_{2}},\left\langle N_{1} * N_{2}\right\rangle^{G_{1} * G_{2}}\right) \cong M\left(G_{1}, M_{1}, N_{1}\right) \oplus M\left(G_{2}, M_{2}, N_{2}\right) .
$$

Proof. Van-Kampen theorem for simplicial groups implies that $K_{1} * K_{2}, L_{1} * L_{2}, R_{1}$. $R_{2}$ and $S_{1} * S_{2}$. are free simplicial resolutions corresponding to $G_{1} * G_{2},\left(G_{1} / N_{1} * G_{2} / N_{2}\right)$, $\left(G_{1} / M_{1} * G_{2} / M_{2}\right)$ and $\left(G_{1} / M_{1} N_{1} * G_{2} / M_{2} N_{2}\right)$, respectively [2]. Since $K_{1}$. and $K_{2}$. are free groups, we have $\left(K_{1} * K_{2}\right)^{a b} \cong K_{1}^{a b} \oplus K_{2}^{a b}$. Hence we have the following commutative diagram

$$
\begin{array}{cccc}
K_{1 .}^{a b} \oplus K_{2 .}^{a b} & \stackrel{\alpha_{1} \oplus \alpha_{2}}{\rightarrow} & L_{1 .}^{a b} \oplus L_{2 .}^{a b} \\
\downarrow & & \downarrow & \downarrow \\
R_{1 .}^{a b} \oplus R_{2}^{a b} & \stackrel{\gamma_{1} \oplus \gamma_{2}}{\rightarrow} & S_{1 .}^{a b} \oplus S_{2 .}^{a b} .
\end{array}
$$

Consequently, $\operatorname{ker}\left(\alpha_{1} \oplus \alpha_{2}, \gamma_{1} \oplus \gamma_{2}\right) \cong \operatorname{ker}\left(\alpha_{1}, \gamma_{1}\right) \oplus \operatorname{ker}\left(\alpha_{2}, \gamma_{2}\right)$ which completes the proof.

Lemma 3.1. Let $G_{1}$ and $G_{2}$ be two groups with $\left(\left|G_{1}^{a b}\right|,\left|G_{2}^{a b}\right|\right)=1$. Then for all $c \geq 1$ the following isomorphism holds

$$
\pi_{n}\left(\frac{K_{1 .} * K_{2 .}}{\gamma_{c}\left(K_{1 .} * K_{2 .}\right)}\right) \cong \pi_{n}\left(\frac{K_{1 .}}{\gamma_{c}\left(K_{1 .}\right)}\right) \oplus \pi_{n}\left(\frac{K_{2}}{\gamma_{c}\left(K_{2 .}\right)}\right)
$$

where $K_{1}$. and $K_{2}$. are free simplicial resolutions of $G_{1}$ and $G_{2}$, respectively.

Proof. First, we consider the following exact sequence which is proved in [10]

$$
1 \rightarrow \operatorname{ker} \phi_{c} \rightarrow \frac{K_{1} * K_{2}}{\gamma_{c}\left(K_{1 .} * K_{2 .}\right)} \rightarrow \frac{K_{1} \times K_{2}}{\gamma_{c}\left(K_{1 .} \times K_{2 .}\right)} \rightarrow 1
$$

Note that ker $\phi_{c}$ satisfies in the following exact sequence

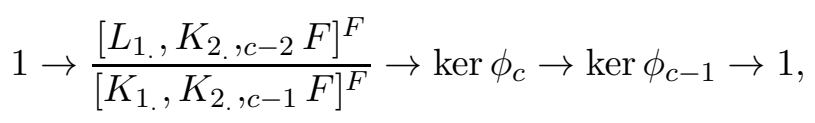

where $F$. $=K_{1} * K_{2}$. Moreover

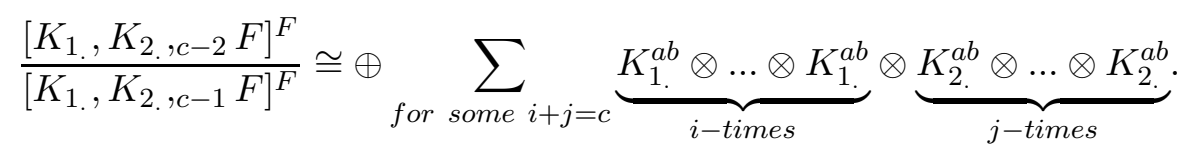

Fix $n$, now by induction on $c$, we show that $\pi_{n}\left(\operatorname{ker} \phi_{c}\right)$ is trivial. For $c=2$, Theorem 3.6 shows that $\operatorname{ker} \phi_{c}=0$. 
Let $c>2$, using Theorem 2.1 we have

$$
\begin{aligned}
\pi_{n}\left(K_{1 .}^{a b} \otimes K_{2 .}^{a b}\right) & \cong \oplus \sum_{i+j=n} \pi_{i}\left(K_{1 .}^{a b}\right) \otimes \pi_{j}\left(K_{2 .}^{a b}\right) \\
& \oplus \sum_{i+j=n-1} \operatorname{Tor}\left(\pi_{i}\left(K_{1 .}^{a b}\right), \pi_{j}\left(K_{2 .}^{a b}\right)\right) \\
& \cong \oplus \sum_{i+j=n} H_{i+1}\left(G_{1}\right) \otimes H_{j+1}\left(G_{2}\right) \\
& \oplus \sum_{i+j=n-1} \operatorname{Tor}\left(H_{i+1}\left(G_{1}\right), H_{j+1}\left(G_{2}\right)\right)=0
\end{aligned}
$$

and

$$
\begin{aligned}
& \pi_{n}(\underbrace{K_{1 .}^{a b} \otimes \ldots \otimes K_{1 .}^{a b}}_{i-\text { times }} \otimes \underbrace{K_{2 .}^{a b} \otimes \ldots \otimes K_{2}^{a b}}_{j-\text { times }}) \cong \\
& \oplus \sum_{r+s=n} \pi_{r}\left(K_{1 .}^{a b} \otimes K_{2 .}^{a b}\right) \otimes \pi_{s}(\underbrace{K_{1 .}^{a b} \otimes \ldots \otimes K_{1}^{a b}}_{i-1-\text { times }} \otimes \underbrace{K_{2 .}^{a b} \otimes \ldots \otimes K_{2}^{a b}}_{j-1-\text { times }}) \\
& \oplus \sum_{r+s=n-1} \operatorname{Tor}\left(\pi_{r}\left(K_{1 .}^{a b} \otimes K_{2 .}^{a b}\right), \pi_{s}(\underbrace{K_{1 .}^{a b} \otimes \ldots \otimes K_{1 .}^{a b}}_{i-1-\text { times }} \otimes \underbrace{K_{2 .}^{a b} \otimes \ldots \otimes K_{2}^{a b}}_{j-1-\text { times }})\right) \\
& =0 .
\end{aligned}
$$

Therefore the induction hypothesis and the exact sequence (3.3) imply that $\pi_{n}\left(\operatorname{ker} \phi_{c}\right)$ is trivial for all $n$.

The exact sequence (3.2) gives rise the following long exact sequence of homotopy groups

$$
\pi_{n}\left(\operatorname{ker} \phi_{c}\right) \rightarrow \pi_{n}\left(\frac{K_{1} * K_{2}}{\gamma_{c}\left(K_{1 .} * K_{2 .}\right)}\right) \rightarrow \pi_{n}\left(\frac{K_{1 .} \times K_{2}}{\gamma_{c}\left(K_{1 .} \times K_{2 .}\right)}\right) \rightarrow \pi_{n-1}\left(\operatorname{ker} \phi_{c}\right) .
$$

Hence the result holds.

Theorem 3.7. Let $\left(G_{i}, M_{i}, N_{i}\right)$ be triples of groups, for $i=1,2$, such that $\left(\left|G_{1}^{a b}\right|,\left|G_{2}^{a b}\right|\right)=$ 1. Then for all $c \geq 1$

$$
M^{(c)}\left(G_{1} * G_{2},\left\langle M_{1} * M_{2}\right\rangle^{G_{1} * G_{2}},\left\langle N_{1} * N_{2}\right\rangle^{G_{1} * G_{2}}\right) \cong M^{(c)}\left(G_{1}, M_{1}, N_{1}\right) \oplus M^{(c)}\left(G_{2}, M_{2}, N_{2}\right) .
$$

Proof. Consider the same notation as the proof of Theorem 3.6 and fix $c$. Applying the homomorphism $\frac{K_{1} * K_{2}}{\gamma_{c}\left(K_{1 .} * K_{2 .}\right)} \rightarrow \frac{K_{1} \times K_{2}}{\gamma_{c}\left(K_{1 .} \times K_{2 .}\right)}$ to the simplicial resolutions of pairs of groups $\left(G_{i}, N_{i}\right)$, for $i=1,2$, we get the following commutative diagram

$$
\begin{array}{ccccccc}
\pi_{n+1}\left(\frac{L_{1} * L_{2}}{\gamma_{c}\left(L_{1 .} * L_{2 .}\right)}\right) & \rightarrow & \pi_{n}\left(\operatorname{ker}\left(\alpha_{1} * \alpha_{2}\right)\right) & \rightarrow & \pi_{n}\left(\frac{K_{1} * K_{2}}{\gamma_{c}\left(K_{1 .} * K_{2 .}\right)}\right) & \rightarrow & \pi_{n}\left(\frac{L_{1} * L_{2}}{\gamma_{c}\left(L_{1 .} * L_{2 .}\right)}\right) \\
\downarrow & & \downarrow & \downarrow & \downarrow & \downarrow & \downarrow \\
\pi_{n+1}\left(\frac{L_{1,} \times L_{2 .}}{\gamma_{c}\left(L_{1 .} \times L_{2 .}\right)}\right) & \rightarrow & \pi_{n}\left(\operatorname{ker}\left(\alpha_{1} \times \alpha_{2}\right)\right) & \rightarrow & \pi_{n}\left(\frac{K_{1} \times K_{2}}{\gamma_{c}\left(K_{1 .} \times K_{2 .}\right)}\right) & \rightarrow & \pi_{n}\left(\frac{L_{1} \times L_{2 .}}{\gamma_{c}\left(L_{1 .} \times L_{2 .}\right)}\right) .
\end{array}
$$

Lemma 3.1 and the Five Lemma imply that $\pi_{n}\left(\operatorname{ker}\left(\alpha_{1} * \alpha_{2}\right)\right) \cong \pi_{n}\left(\operatorname{ker}\left(\alpha_{1}\right)\right) \oplus \pi_{n}\left(\operatorname{ker}\left(\alpha_{2}\right)\right)$. Similarly for the pair of groups $(G / M, M N / M)$ we have $\pi_{n}\left(\operatorname{ker}\left(\gamma_{1} * \gamma_{2}\right)\right) \cong \pi_{n}\left(\operatorname{ker}\left(\gamma_{1}\right)\right) \oplus$ $\pi_{n}\left(\operatorname{ker}\left(\gamma_{2}\right)\right)$. With a similar argument for the exact sequence (3.1) we have the following commutative diagram

$$
\begin{aligned}
& \rightarrow \pi_{n}\left(\operatorname{ker}\left(\alpha_{1} * \alpha_{2}\right), \operatorname{ker}\left(\gamma_{1} * \gamma_{2}\right)\right) \rightarrow \pi_{n}\left(\operatorname{ker}\left(\alpha_{1} * \alpha_{2}\right)\right) \rightarrow \pi_{n}\left(\operatorname{ker}\left(\gamma_{1} * \gamma_{2}\right)\right) \\
& \rightarrow \quad \pi_{n}\left(\operatorname{ker}\left(\alpha_{1}, \gamma_{1}\right), \operatorname{ker}\left(\alpha_{2}, \gamma_{2}\right)\right) \quad \rightarrow \quad \pi_{n}\left(\operatorname{ker}\left(\alpha_{1}, \alpha_{2}\right)\right) \quad \rightarrow \quad \pi_{n}\left(\operatorname{ker}\left(\gamma_{1}, \gamma_{2}\right)\right) \text {. }
\end{aligned}
$$


Therefore we can prove that $\pi_{n}\left(\operatorname{ker}\left(\alpha_{1} * \alpha_{2}, \gamma_{1} * \gamma_{2}\right)\right) \cong \pi_{n}\left(\operatorname{ker}\left(\alpha_{1}, \gamma_{1}\right)\right) \oplus \pi_{n}\left(\operatorname{ker}\left(\alpha_{2}, \gamma_{2}\right)\right)$. Hence for $n=1$, we have $M^{(c)}\left(G_{1} * G_{2},\left\langle M_{1} * M_{2}\right\rangle^{G_{1} * G_{2}},\left\langle N_{1} * N_{2}\right\rangle^{G_{1} * G_{2}}\right) \cong M^{(c)}\left(G_{1}, M_{1}, N_{1}\right) \oplus M^{(c)}\left(G_{2}, M_{2}, N_{2}\right)$.

Theorem 3.8. Let $M^{(1)}(G, N), M^{(1)}(G / N)$ and $H_{3}(G / N)$ be torsion groups. Then $M^{(c)}(G, N)$ is also a torsion group, for all $c \geq 2$.

Proof. Let $K$. and $L$. be free simplicial resolutions of $G$ and $G / N$, respectively. Consider the epimorphisms $\frac{K .}{\gamma_{n}(K .)} \stackrel{\alpha_{n}}{\rightarrow} \frac{L}{\gamma_{n}(L .)}$ and $\frac{\gamma_{n}\left(K_{.}\right)}{\gamma_{n+1}(K .)} \stackrel{\alpha_{n} / \alpha_{n+1}}{\rightarrow} \frac{\gamma_{n}\left(L_{.}\right)}{\gamma_{n+1}(L .)}$. By [4, Claim 2] $\pi_{1}\left(\gamma_{n}\left(K_{.}\right) / \gamma_{n+1}\left(K_{.}\right)\right)$is a torsion group. In [10] the authors obtained the following exact sequence

$$
\pi_{2}\left(\frac{\gamma_{n}\left(L_{.}\right)}{\gamma_{n+1}(L .)}\right) \rightarrow \pi_{1}\left(\operatorname{ker} \alpha_{n} / \alpha_{n+1}\right) \rightarrow \pi_{1}\left(\frac{\gamma_{n}\left(K_{.}\right)}{\gamma_{n+1}(K .)}\right) .
$$

Note that $\pi_{1}\left(\operatorname{ker} \alpha_{n} / \alpha_{n+1}\right)$ satisfies in the following exact sequence

$$
\pi_{1}\left(\operatorname{ker} \alpha_{n} / \alpha_{n+1}\right) \rightarrow M^{(n+1)}(G, N) \rightarrow M^{(n)}(G, N)
$$

With a similar argument of [4, Claim 2] we can get the isomorphism $\pi_{2}\left(L^{a b} \otimes Q\right) \cong$ $H_{3}(G / N) \otimes Q=0$ which leads to $\pi_{2}\left(\gamma_{n}\left(L_{.}\right) / \gamma_{n+1}(L).\right) \otimes Q \cong 0$. Therefore $\pi_{2}\left(\gamma_{n}(L.) / \gamma_{n+1}\left(L_{.}\right)\right)$ is a torsion group. Thus (3.4) implies that $\pi_{1}\left(\operatorname{ker} \alpha_{n} / \alpha_{n+1}\right)$ is a torsion group. Hence the result follows by induction from (3.5).

Theorem 3.8 can be extended to triples of the groups with the similar argument.

Theorem 3.9. Let $M^{(1)}(G, M, N), M^{(1)}(G), H_{3}(G / N), H_{3}(G / M)$ and $H_{4}(G / M N)$ be torsion groups. Then $M^{(c)}(G, M, N)$ is a torsion group, for all $c \geq 1$.

Proof. A detailed proof is quite lengthy, so we give a sketch of proof. Consider the following commutative diagrams which induce from the diagram of the free simplicial resolutions of triple of groups $(G, M, N)(1.1)$
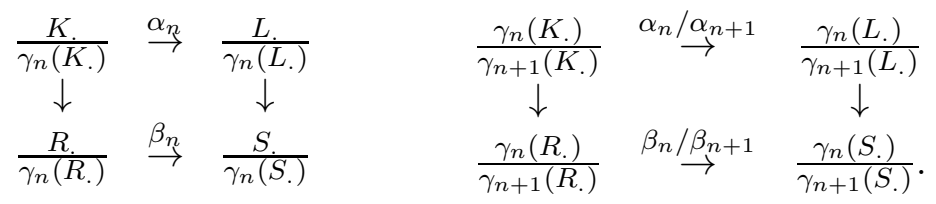

With a similar argument of [11], we can obtain the following exact sequence

$$
\pi_{1}\left(\operatorname{ker}\left(\alpha_{n} / \alpha_{n+1}, \beta_{n} / \beta_{n+1}\right)\right) \rightarrow M^{(n+1)}(G, M, N) \rightarrow M^{(n)}(G, M, N)
$$

which satisfies in exact sequence

$$
\pi_{2}\left(\operatorname{ker} \beta_{n} / \beta_{n+1}\right) \rightarrow \pi_{1} \operatorname{ker}\left(\left(\alpha_{n} / \alpha_{n+1}, \beta_{n} / \beta_{n+1}\right)\right) \rightarrow \pi_{1}\left(\operatorname{ker} \alpha_{n} / \alpha_{n+1}\right) .
$$


Moreover, $\pi_{2}\left(\operatorname{ker} \beta_{n} / \beta_{n+1}\right)$ fits in the following exact sequence

$$
\pi_{3}\left(\frac{\gamma_{n}(S .)}{\gamma_{n+1}(S .)}\right) \rightarrow \pi_{2}\left(\operatorname{ker} \beta_{n} / \beta_{n+1}\right) \rightarrow \pi_{2}\left(\frac{\gamma_{n}(R .)}{\gamma_{n+1}(R .)}\right) .
$$

By Theorem 3.8, we have $\pi_{2}\left(\operatorname{ker} \alpha_{n} / \alpha_{n+1}\right)$ and $\pi_{2}\left(\frac{\gamma_{n}(R .)}{\gamma_{n+1}(R .)}\right)$ are torsion groups. Hence the result is proved if we show that $\pi_{3}\left(\frac{\gamma_{n}(S .)}{\gamma_{n+1}(S .)}\right)$ is a torsion group.

With a similar argument of the proof of Theorem 3.8, we can prove that if $H_{4}(G / M N)$ is a torsion group, then so is $\pi_{3}\left(\frac{\gamma_{n}\left(S_{\text {. }}\right)}{\gamma_{n+1}\left(S_{\text {. }}\right)}\right)$.

\section{References}

[1] J. Barja, C. Rodreguez. Homology groups $H_{n}^{q}(-)$ and eight-term exact sequences. Cahiers de Topologie et Géométrie Differéretielle catégoriques, 1990, 31(2): 91-120.

[2] J. Burns, G. Ellis. On the nilpotent multipliers of groups. Math. Z., 1997, 226: 405-428.

[3] E.B. Curtis. Simplicial homotopy theory. Advances in Math., 1971, 6: 107-209 .

[4] G. Ellis. A magnus-witt type isomorphism for non free groups. Georgian Mathematical Journal, 2002, 9(4): 703-708.

[5] G. Ellis. The Schur multiplier of a pair of groups. Applied Categorical Structures, 1998 6: $355-371$.

[6] L. Franco. Simplicial deirvation, higher Bear invariant and homology of crossed modules. Communications in Algebra, 1998, 26(4): 1125-1139.

[7] P.G. Georss, J.F. Jardine. Simplicial Homotopy Theory. Progress in Math., 174 Bikhauser, Boston-Basel-Berlin, 1999 .

[8] H. Hopf. Fundamental gruppe und zweite Bettische Gruppe. Comment. Math. Helvetici, 1942, 14: 257-309.

[9] H.N. Inassaridze. Homotopy of pseudosimplicial groups and nonabelian derived functors. Sakharth. SSR Mecn. Akad. Moambe, 1974, 76: 533-536.

[10] Z. Vasagh, H. Mirebrahimi, B. Mashayekhy. On Baer Invariants of groups with topological approach. arXiv:1010.0291.

[11] Z. Vasagh, H. Mirebrahimi, B. Mashayekhy. On Baer invariants of pairs of groups. arXiv:1106.1278 